\title{
Utilization of Learners' Metacognitive Experiences to Monitor Learners' Cognition States in e-Learning Platforms
}

\author{
Othmar Mwambe and Eiji Kamioka
}

\begin{abstract}
Optimal learning environment highly depends on aptitude treatment interaction. Even though ICT (Information and Communication Technologies) advancement supports knowledge sharing platforms including e-learning and multimedia, designing of learning content in such platforms relies on the aptitude treatment interaction to sustain a learner-centric environment. However, achieving the optimal learning environment in e-learning platform is still a challenge due to variation of learner's skills and abilities to both learning process and learning content. This makes difficulties in monitoring learner's cognition states and quality of learning content. To overcome the difficulties, in this study, a learner-centric metacognitive experiences based approach has been proposed (e-learning Prior Knowledge Assessment System-ePKAS), ePKAS is supporting the detection and evaluation of learners' prior knowledge profiles in turn to enable monitoring of learners' cognition states and adaptation of cognitive states in to e-learning platforms based on visual contact. The study has investigated students' reactions to multimedia content based on their past experiences. The results show that students respond more attentively and accurately $(93 \%)$ to the learning content that is closely related to their past experiences. Scope of this study based on visual contact in order to support the involvement of people with hearing impairment in e-learning platforms.
\end{abstract}

Index Terms-E-learning, cognition, aptitude treatment interaction, metacognitive experiences, memory retrieval, multimedia, long-term memory, cognitive structure.

\section{INTRODUCTION}

Dramatic growth of Information and Communication Technologies (ICT) has contributed to the development of e-learning platforms, which are reliable tools for knowledge sharing in a modern education system. Technology advancement has immensely improved e-learning platforms to provide learners with user-friendly environment [1] and in turn to support optimal learning. In e-learning platforms, Learning process and multimedia rely mostly on learners' self- regulation. However, monitoring learners' reactions to the learning contents and evaluating the quality of such contents in e-learning platforms have been challenges [2]. This is because of variation of learners' abilities and skills to learning contents. Such variation hinders not only an optimal learning but also fades away involvement of deaf individuals in e-learning platforms [3].

To overcome such challenges, a learner-centric

Manuscript received June 20, 2018; revised January 18, 2019.

The authors are with the Shibaura Institute of Technology, Japan (e-mail: mg17007@shibaura-it.ac.jp, kamioka@ shibaura-it.ac.jp). metacognitive experiences based approach has been introduced to support detection and evaluation of learners' prior knowledge profiles in turn to enable monitoring of learners' cognition states and adaptation of cognitive states in to e-learning platforms. Also the scope of this study focuses on visual contact so as to enable deaf students to participate in e-learning. This study assumes that learners' metacognitive experiences influence learner's attitude to multimedia learning content.

Multimedia content plays a vital role in e-learning platforms as the dissemination of information in e-learning platforms uses multimedia components such as text, audio, videos, animation and interactive content. Multimedia content has significant effects on our visual perception [4] and learning process [5]. Metacognitive experiences and memory retrieval play an important role in learning process, whereby visual objects stimulate information retrieval of our past experiences from long-term memory [6].When difficult retrieving is experienced, the study refers to such cognitive state as metacognitive experiences [7].

Cognitive structure and Multimedia - Short-term memory and long-term memory are core components of our cognitive structure that define functional connectivity of our brain system and cognitive states. Temporary storage and manipulation of information is defined by short-term memory, commonly referred as working memory. Permanent storage of information that we acquire throughout life time is handled by long-term memory. Working memory is composed of four major elements, central executive, visual spatial sketchpad, phonological loop and episodic buffer [8]. When we are subjected to perform some tasks in multimedia environment, our visual perception automatically makes use of episodic buffer to recall our previous experiences.

This paper is organized as follows: Related work will be stated in Section II. Proposed system will be elaborated in Section III. Verification experiment and the results will be explained in Section IV. In Section V, discussions based on the experimental results will be done, and finally conclusion and future perspectives will be stated in Section VI.

\section{RELATED WORK}

Adaptation of cognitive states into learning platforms has been an interest of many researchers. Johansson, R., \& Johansson $\mathrm{M}$. investigated the role of eye movements during information retrieval using eye tracking; they found out that eye movement plays an important role in memory retrieval [6]. Daniel, K. N., \& Kamioka, E.adapted concentration 
cognitive state into distant learning platforms by using eye tracking technology; they proposed a system that supports detection of learner's concentration [9]. Endler, A., et Al. investigated the possibilities of adapting motivation states into e-learning platforms and identified the existing difficulties that result from the lack of cogntive states' adapative features in e-learning platforms [10]. Mwambe, O., \& Kamioka, E. investigated the correlation between visual spatial attention and long-term memory using electroencephalogram (EEG); they proposed a brain computer interface approach to support aptitude treatment interaction in e-learning platforms [11].

In this study, a learner-centric metacognitive experience based approach namely; e-learning prior knowledge assessment system is proposed. The proposed approach can support detection and evaluation of learners' prior knowledge profiles. After finding the correlation between visual spatial attention and long-term memory in our previous study [11], then we decided to conduct this verification study that imitates real e-learning environment. Unlike our previous study, the stimuli order in this study was randomized to avoid presentation-order effect, and thus, imitated the real e-learning environment. Scope of this study focuses only on visual contact as an extension of our movement [12] towards fully involvement of people with hearing impairment in e-learning platforms

\section{PROPOSED APPROACH}

As an extension of our previous work [11], e-learning Prior Knowledge Assessment system (ePKAS) is designed to support instructors to understand their learners' prior knowledge profiles level with respect to the new learning materials and instruction.

\section{A. Design Requirements}

The proposed system is to let instructors understand their students' prior knowledge profiles level so as to adjust their instructional strategies and teaching content. Cognitive states define learners' mental state and in turn it determines learners' level of understanding the learning contents and knowledge.It is very important to assess prior knowledge profiles of learners so as to determine instructional approach and to evaluate teaching contents. Thefore, to achieve that goal system has to meet the following reqirement:

1) Enable learners and instructor to interact online

System must enable instructors and learners to interact in learning management system (LMS) module. LMS consists of Multimedia content, Instruction module, Assessment module and Recommendation module

2) Enable acquisition of learners' brain biological information

System must include learners' Brain information acquisition module eg. EEG sensor that can enable observation of brain changes in time domain basis.

1) Detect learners' metacognitive experiences

Based on acquired biological information, system must be able to detect learners' difficult retrieving cognitive state that is referred by study as metacognitive experience.

2) Identify learners' prior knowledge level
System must be able to recommend learners 'prior knowledge profiles.

\section{B. Proposed System}

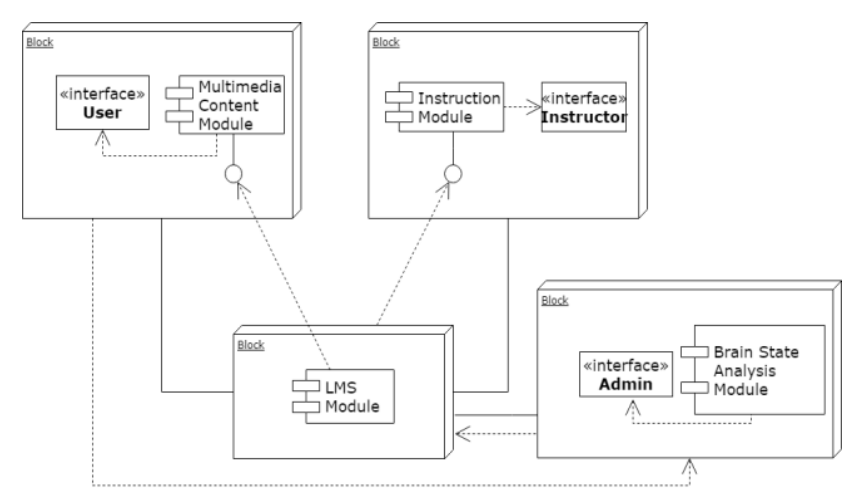

Fig. 1. E-learning prior knowledge assessment system (ePKAS).

Fig. 1 shows the diagram of proposed system (ePKAS). It consists of four core modules: Multimedia Content module, Instruction module, and Learning Management Module (LMS) and Brain State Analysis (BSAM) Module. The four modules are operating in parallel and depending on one another. However, main function class is managed by LMS module. LMS module provides interfaces that enable learners, system administrator and instructors to interact online. As many other e-learning platforms, all ePKAS operations will be centralized in LMS but ePKAS does have additional module (BSAM) that gives it extra intelligent capacity to evaluate learners' brain cognitive state. Thus, in this section only BSAM will be explained in details as it carries contribution of the study.

Multimedia Content Module - provides learners with access to the interactive learning environment using multimedia content. While are interacting with multimedia content, learners' brain biological information is intuitively collected using brain sensor embedded in the wearable device

Instruction Module - provides instructors and learning content designers with access to the instruction and design interface whereby instructors can be able to set rules and conditions that govern the displayed multimedia content for the evaluation purpose and learners' performance prediction analysis. Base on recommendation from BSAM module ePKAS should be able to make auto adjustment of the displayed Multimedia content in real time

Brain State Analysis Module (BSAM) - obtains learners' brain cognitive states data and analyses to support the efficiency of the evaluation process. Detected frequency is analyzed using Fast Fourier Transform (FFT) and result is sent to the LMS assessment module to assist assessment process. Base on obtained test result and detected frequency band the system recommends learners' prior knowledge profile level to the instruction module for customization of multimedia content.

Fig. 2 shows the ePKAS-users interaction model. User interface enables learners and instructors to interact online whereby learners' brain biological information is intuitively collected using brain sensor that is embedded in his/her earphone device. Detected frequency is analyzed using Fast Fourier Transform (FFT) and result is sent to the LMS assessment module. Base on obtained detected frequency 
band the system recommends learners' prior knowledge profile level to the instructor using system recommendation module.

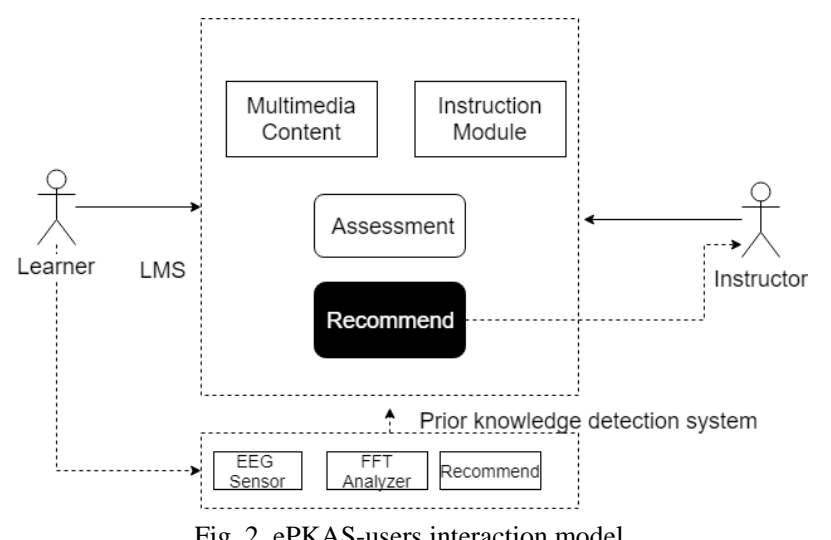

Base on the threshold observed in our previous study [11] ePKAS is able to provide smart decision making process. Once signal is received from the sensor then system will convert it into FFT Data for analysis. If the data fits threshold range then the system is recommending learners' cognitive state unless otherwise the process will be repeated to acquire optimum value.

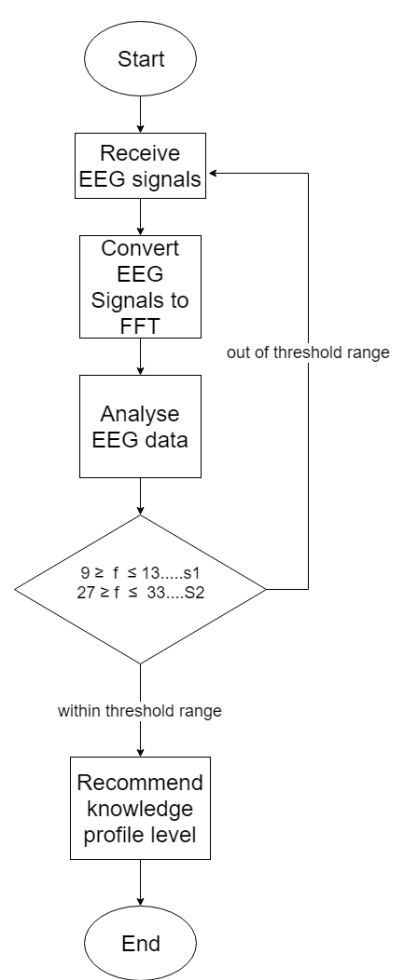

Fig. 3. ePKAS algorithm.

Once signal is received from the sensor then system will convert it into FFT Data for analysis. If the data fits threshold range then the system is recommending learners' cognitive state unless otherwise the process will be repeated to acquire optimum value. If the the data falls withing the defined range then ePKAS is able to determine the matching level (prior knowledge) inturn recommends customization approach.

Fig. 3 shows proposed ePKAS algorithm, it is defined by two states with respect to the obtained threshold from the experiment 1 , the states ( $\mathrm{s} 1$ and $\mathrm{s} 2$ ) are defined base on freguency range detected during the task execution.S1 defines cognitive state when the learning content matches learner's prior knowledge profile while S2 defines the cognitive state when learning content does not match learner's prior knowledge profile.

\section{VERIFICATION EXPERIMENT}

To verify the feasibility of our proposed approach in a real e-learning environment, an experiment was conducted at Shibaura Institute of Technology (SIT), involving six subjects. The experiment was set to imitate an e-learning environment.

\section{A. Demographic Information of Subjects}

All the subjects (five males and 1 female)were students belonging to Department of Computer Science, at SIT, aged between 21- 30 years old; Three master's, one $\mathrm{PhD}$ and two undergraduate students.

\section{B. Experiment Set up}

Subjects were subjected to multimedia content that consisted of two types of images and multiple choice tests. The images were of two categories, familiar and unfamiliar. Familiar consisted of images of computer devices while unfamiliar consisted of images of unfamiliar devices different from subjects' field of expertise. Subjects were instructed to identify the names of the displayed devices by responding to the online tests. The images (familiar and unfamiliar) were randomly displayed on the PC monitor of the computer and the subjects were instructed to address all the questions of the online tests. There were 40 images in total and each displayed image was followed by its corresponding test. Response time and accuracy were recorded using the system that was developed for the experiment. Fig. 4 shows the procedure of the experiment.

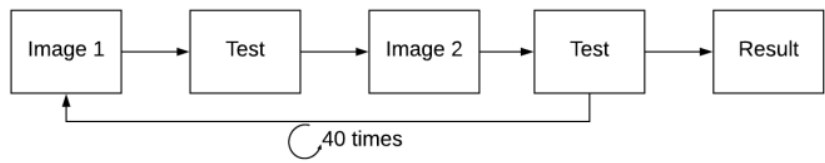

Fig. 4. Experiment procedure.

\section{Results}

Fig. 5 Illustrates the average response time of the online test for each subject. The figure shows that the average response time to questions on familiar devices is much shorter than the average response time to questions on unfamiliar ones. Subjects spent more time with less accuracy on tests corresponding with the images of unfamiliar devices. On average, the subjects spent 1.6 seconds on questions corresponding with the familiar information and 2.5 seconds on unfamiliar information, respectively.

Fig. 6. It illustrates the accuracy to the questions for each subject. The figure shows that all the subjects addressed questions related to images of familiar devices with higher accuracy than unfamiliar ones. They correctly managed to identify names of familiar images within a short period of time compared with unfamiliar ones where more time was spent with less accuracy. On average, the subjects addressed familiar information with accuracy of $93 \%$ and unfamiliar 
information with accuracy of $7 \%$, respectively.

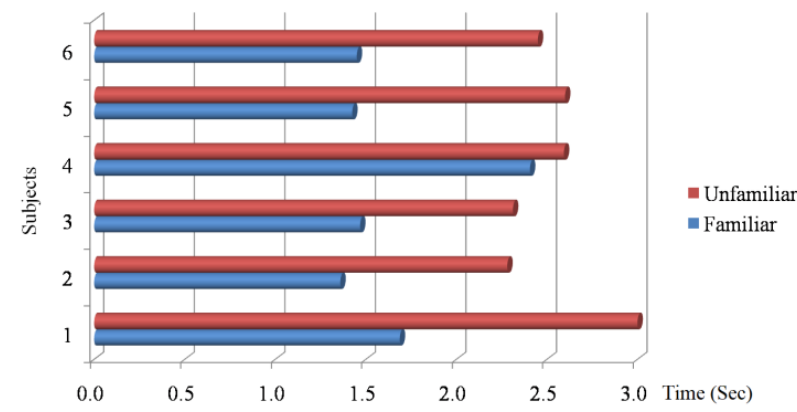

Fig. 5. Response time to questions of online tests.

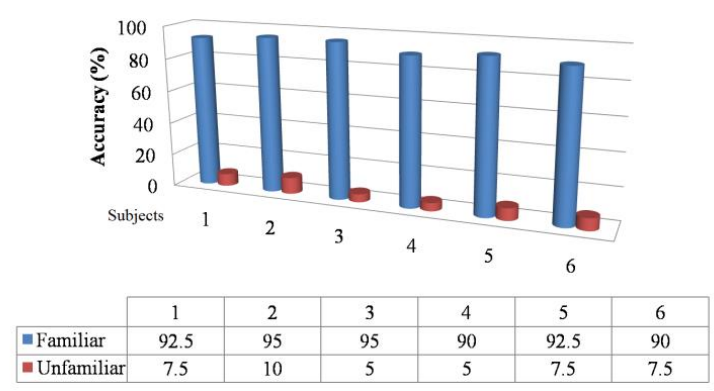

Fig. 6. Accuracy on questions of online tests.

\section{DISCUSSION}

Short response time and high accuracy on questions related to the names of familiar devices show easy retrieval of information and students' higher motivation when subjected to the learning content that matches their past experiences. However, students' long response time on questions related to the images of unfamiliar devices and poor accuracy show difficulties of information retrieval when subjected to the unfamiliar information that does not match their past experiences.

Low accuracy and long response time on questions related with unfamiliar information also reflects students' poor understanding and low motivation to the learning content. Thus, detecting learners' metacognitive experiences during learning process may help to determine students' motivation level and cognition. However, to what extent has a learner understood the learning content is an open research area for future studies on cognitive load. To this extent the study can only contribute much to the prior knowledge assessment.

Even though response time and accuracy have been found to be reliable parameters, however, variation exists from one subject to another cannot be well explained by relying on long-term memory alone. Hence, more effort should be taken to explore episodic buffer so as to derive clear relation between the cognitive load and such parameters. Clear means of cognitive measurement will not only provide much support on learners' self-regulation and monitoring of students' cognition states during learning process but also prediction of learners' performance.

\section{CONCLUSION AND FUtURE PERSPECTIVES}

This study investigated learner's attitude to multimedia learning content and proposed a learner-centric metacognitive experiences based approach to support detection and evaluation of learners' prior knowledge in turn to enable monitoring of learners' cognition states and adaptation of cognitive states in to e-learning platforms. The results show that metacognitive experiences can be useful information to determine learners' cognition states and presentation order effect had less impact on subjects' reaction to observed stimuli. We are looking forward to exploring further the influence of individual differences on working memory (episodic buffer) performances so as to be able to propose brain-computer interfaces based approach for cognitive load assessment and adaptive navigation support for e-learning platforms.

\section{REFERENCES}

[1] S. Y. Nof, J. Ceroni, W. Jeong, and M. Moghaddam, "E-learning and e-training," Revolutionizing Collaboration through e-Work, e-Business, and e-Service, pp. 357-390, Springer, Berlin, Heidelberg, 2015.

[2] D. Seth, E. Marshman, and C. Singh, "The challenge of engaging all students via self-paced interactive e-learning tutorials for introductory physics," 2016.

[3] B. Fabrizio, C. S. Bianchini, and M. Marsico, "UX," in Proc. International Conference on Universal Access in Human-Computer Interaction, pp. 221-232, Springer, Cham, 2014.

[4] R. Zheng, "Cognitive effects of multimedia learning," IGI Global, 2008.

[5] N. Hossein and A. Shahid, "The effect of PowerPoint presentations on student learning and attitudes," Global Perspectives on Accounting Education, vol. 2, no. 53, 2005.

[6] J. Roger and M. Johansson, "Look here, eye movements play a functional role in memory retrieval," Psychological Science, vol. 25, no. 1, pp. 236-242, 2014.

[7] P. Eleonora, "The concept and instruction of metacognition," Teacher Development, vol. 7, no. 1, pp. 9-30, 2003.

[8] A. D. Baddley, "The episodic buffer: A new component of working memory?" Trends in Cognitive Sciences, vol. 4, no. 11, pp.417-423, 2000.

[9] K. N. Daniel and E. Kamioka, "Detection of learner's concentration in distance learning system with multiple biological information," Journal of Computer and Communications, vol. 5, no. 04, p. 1, 2017.

[10] A. Endler, G. D. Rey, and M. V. Butz, "Towards motivation-based adaptation of difficulty in e-learning programs," Australasian Journal of Educational Technology, vol. 28, no. 7, 2012.

[11] O. O. Mwambe and E. Kamioka, "EEG cognition detection to support aptitude-treatment interaction in e-learning platforms," IEEE, 2018.

[12] O. O. Mwambe, S. S. Nathan, T. Nguyen-Duc, and E. Kamioka, "FFCDH: Solution to enable face-to-face conversation between deaf and hearing people," Journal of Computer and Communications, vol. 6, pp. 1-14, 2018.

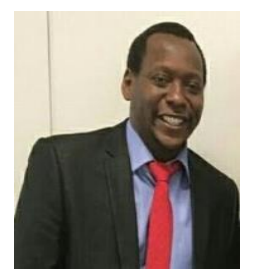

Othmar Othmar Mwambe is a graduate student at Shibaura Institute of Technology (SIT). He studied computer engineering, specialized in computer systems and networks at Kharkiv National University of Radio-electronics (KNURE), Ukraine.

Before joining SIT, he was working for Tumaini University, Dar es salaam Institute of Technology (DIT) as instructor and National Institute of Informatics (NII), Graduate University for Advanced Studies (SOKENDAI) as a research student. His current research interests include Cyber security, Management Information Systems (MIS), Ubiquitous computing and Brain Computer Interfaces (BCI).

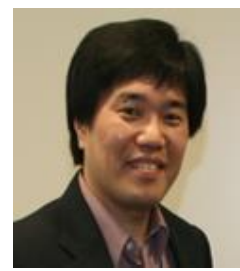

Eiji Kamioka is professor of electrical engineering and computer science, deputy president at SIT, his B.S, M.S and D.S degrees in Physics from Aoyama Gakuin University.

Before joining SIT, he was working for SHARP Communication Laboratory, Institute of Space and Astronautical Science (ISAS) as JPSP research fellow and National Institute of Informatics (NII) as assistant professor. His current research interests include mobile multimedia communications and ubiquitous computing. 